\title{
On Direct Observation of Diffusion by Aberration-Corrected HREM
}

\author{
J.T. McKeown ${ }^{*}$, Y.-W. Kim ${ }^{* *}$, W.T. Petuskey ${ }^{* * *}$, M. O’Keeffe ${ }^{* * *}$, U. Weierstall ${ }^{*}$, \\ and J.C.H. Spence \\ * Department of Physics, Arizona State University, Tempe, AZ 85287 \\ ** School of Materials Science, Seoul National University, Seoul 151-742, Korea \\ *** Department of Chemistry, Arizona State University, Tempe, AZ 85287
}

Renewable energy sources such as wind and solar energy, despite their abundance and availability, are variable and uncontrolled [1]. A continuous supply of clean energy therefore requires a significant expansion in energy storage and conversion technologies. Solid electrolytes enable technologies that can both convert (fuel cells) and store (batteries) electrical energy. The ability of a solid electrolyte to conduct ions will depend largely on microstructural defects in the material. Line and planar defects can scatter ions or build up a space-charge layer to affect ionic conductivity. More fundamentally, the diffusion of ions will be closely correlated to point-defect diffusion. Our aim is to conduct dynamic in-situ experiments to study the role of defects in a solid electrolyte by direct observation of atomic motion at and along defects using aberration-corrected HREM. Since conductivity $(\sigma)$ is proportional to jump frequency $(\Gamma)$, experiments that directly observe jump frequency will provide fundamental insight into charge-transport processes in these materials.

In order to directly observe diffusion, 1) the atomic jump frequency must be sufficiently low, and 2) there must be a discernible contrast change in the high-resolution image when atoms move between adjacent atomic columns. The jump frequency (for vacancy mechanisms) is given by:

$$
\sigma \propto \Gamma=v_{z} X_{v} \exp \left[\frac{-E_{a}}{k T}\right]
$$

where $v$ is an attempt frequency, $z X_{v}$ is a concentration of vacant nearest-neighbor sites, and $E_{a}$ is an activation energy. Jump frequencies are typically $10^{3} \mathrm{~s}^{-1}$ at liquid-nitrogen temperatures to $10^{10} \mathrm{~s}^{-1}$ at room temperature for $\mathrm{Na} \beta$-alumina [2]). It is well known [3-5] that the sodium ion content in $\mathrm{Na} \beta$-alumina can be replaced with divalent cations such as $\mathrm{Ca}^{2+}, \mathrm{Sr}^{2+}, \mathrm{Ba}^{2+}, \mathrm{Zn}^{2+}, \mathrm{Cd}^{2+}$, and $\mathrm{Pb}^{2+}$, yielding higher activation energies and lower diffusion rates (Fig. 1). If only a partial ion exchange is performed, the larger atomic specie will provide a contrast difference in high-resolution images, which will allow the in-situ observation of cation diffusion in these solid electrolytes. The application of HRTEM and HR-STEM (Fig. 2) will be discussed, as well as complications that arise from radiation damage [6]. We are particularly interested in the possibility of correlated motion among the $\mathrm{Pb}^{2+}$ ions in their "liquid" sheets within the conduction planes $[7,8]$.

\section{References}

[1] X. Lu et al. J. Power Sources 19520102431.

[2] A.R. West in Solid State Electrochemistry, P.G. Bruce (ed.), Cambridge University Press, Cambridge, 1995. 
[3] Y.-F. Yu Yao, and J.T. Kummer. J. Inorg. Nucl. Chem. 2919672453.

[4] B. Dunn et al. Solid State Ionics 51981203.

[5] R. Seevers et al. J. Solid State Chem. 501983146.

[6] R. Hull et al. Solid State Ionics 9\&10 1983181.

[7] S. Jacobsen et al. J. Chem. Phys. 7819834154.

[8] This work was supported by DOE Contract No. DE-FG03-02ER45996.

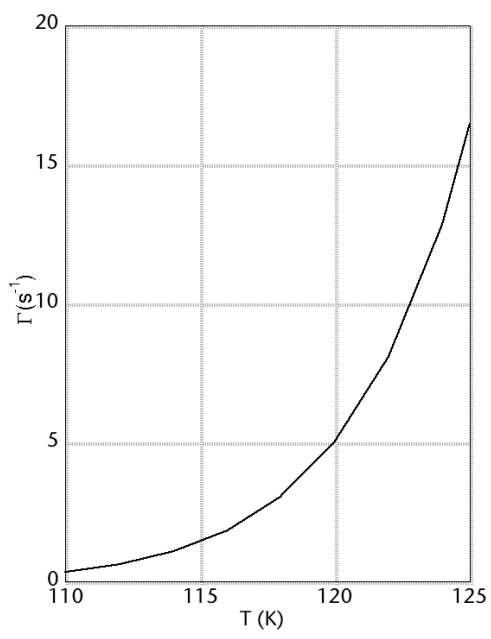

FIG. 1. Plot of the jump frequency, $\Gamma$, as a function of temperature for $\mathrm{Pb}^{2+}$ in $\beta^{\prime \prime}-$ alumina. An attempt frequency, $v$, of $10^{13} \mathrm{~s}^{-1}$ and an activation energy of $0.3 \mathrm{eV}[1]$ have been used. A jump frequency of $\approx 1 \mathrm{~s}^{-1}$ is achieved at $\approx 110-115 \mathrm{~K}$. At liquid nitrogen temperatures, the jump frequency is $\approx 10^{-7} \mathrm{~s}^{-1}$.

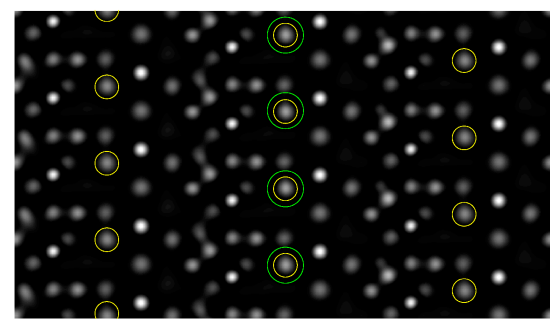

(a)

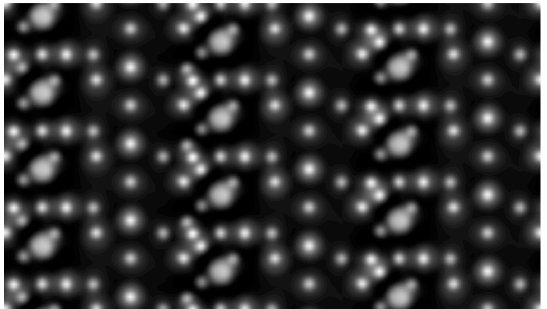

(b)

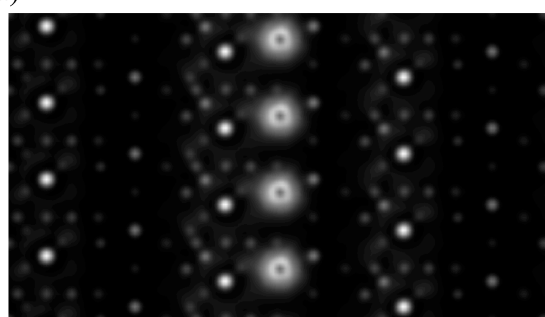

(c)

FIG. 2. (a) HRTEM, (b) bright-field HR-STEM, and (c) HAADF HR-STEM multislice image simulations for the [10 10 ] zone axis of $\beta^{\prime \prime}$-alumina using an aberration-corrected microscope operating at $200 \mathrm{keV}$. The conduction planes in the structure are indicated in (a), where yellow circles show the positions of $\mathrm{Na}^{+}$ions and green circles show columns in which a single $\mathrm{Pb}^{2+}$ ion has been substituted at $\approx 65 \AA$ through the thickness of $\approx 120 \AA$. $\mathrm{The}^{2+} \mathrm{Pb}^{2+}$ ions are evident in the HAADF HR-STEM image. 